\title{
STREAK CAMERA MEASUREMENTS OF THE LONGITUDINAL DISTRIBUTION WITH MULTIPLE BUNCHES IN CESR*
}

\author{
$\underline{\text { R. Holtzapple }}{ }^{+}$, M. Billing, and D. Hartill \\ Laboratory of Nuclear Studies, Cornell University, Ithaca, NY 14853
}

\begin{abstract}
The Cornell Electron-Positron Storage Ring (CESR) has a longitudinal dipole-coupled-bunch instability that limits the total amount of current that can be stored in the ring. As a result, it is one of the major limitations for higher luminosity [1]. This paper reports the results from a streak camera used to measure the dependence of the bunch's distribution on current and accelerating RF voltage for multiple bunches in CESR, as well as the effects of the longitudinal instability on the bunch distribution. Measurements of the bunch's longitudinal distribution at CESR, with multiple bunches present in the ring, help give an understanding of the nature of the longitudinal instability.
\end{abstract}

\section{MULTIPLE BUNCH OPERATION AT CESR}

Multiple bunches refers to more than one bunch of electrons or positrons being present in CESR at one time. Multiple bunches in CESR are necessary to achieve high luminosity. Under present high-energy physics colliding beam conditions, there are nine nearly evenly spaced bunch trains, with up to five bunches in each train, for a total of 45 electron and 45 positron bunches (Figure 1). The bunches are separated at crossing points other than the interaction region by vertical and horizontal electrostatic separators. The separators are used to make a "pretzel" orbit to insure separation between bunch crossings at locations other than the interaction region. Different distributions of bunches in the trains are observed to have different dipole-coupled bunch instability thresholds.

$$
\text { Train \#1 }
$$
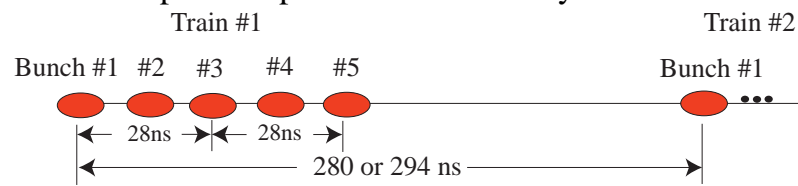

Figure 1. There are nine trains of bunches in CESR and each train can have up to five bunches present (five bunches per train are shown in this figure). Each train is separated by 280 or $294 \mathrm{~ns}$ and the bunch spacing in the train are in multiples of $14 \mathrm{~ns}$.

\section{DATA ANALYSIS}

Measurements of the CESR longitudinal bunch distribution were made with a 500-femtosecond resolution Hamamatsu streak camera. A description of the camera and experimental set-up is described in detail elesewhere [2].

To determine the bunch length, the longitudinal profiles of the beam distribution are fit to an asymmetric Gaussian function with a constant background given by

$$
\mathrm{I}(\mathrm{z})=\mathrm{I}_{0}+\mathrm{I}_{1} \exp \left\{-\frac{1}{2}\left(\frac{(\mathrm{z}-\overline{\mathrm{z}})}{(1+\operatorname{sgn}(\mathrm{z}-\overline{\mathrm{z}}) \mathrm{A}) \sigma_{\mathrm{z}}}\right)^{2}\right\}
$$

where $I_{0}$ is the pedestal, and $I_{1}$ is the peak of the asymmetric Gaussian. The term $\operatorname{sgn}(z-\bar{z}) A$ is the asymmetry factor that parameterizes the shape of this Gaussian. The mean rms width, $\sigma_{z}$, and asymmetry factor, A, from each experiment, are used to examine the pulse-to-pulse fluctuations in the beam distribution. Each distribution is fit to the mean rms width and asymmetry factor and the area and mean position of the distribution can vary. The difference, (or residuals) between the fit and the data will show variations between an average distribution and individual data acquisitions.

\section{STABLE BUNCH MEASURMENTS}

The electron bunch distribution, as a function of current, was measured with nine trains of two bunches per train. The separation between the two bunches, in a train, was $42 \mathrm{~ns}$. The wiggler magnets were closed during this measurement. The mean and the root mean error for the bunch width and asymmetry factor, at each current setting, is plotted in Figures 2 (a) and (b).
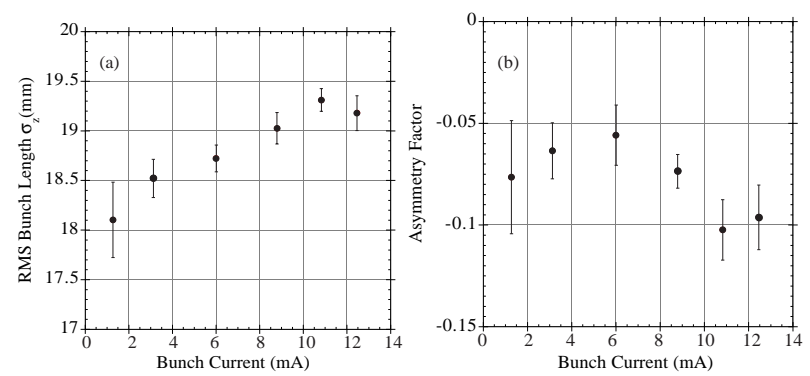

Figure 2. The electron (a) bunch length as a function of current with 18 bunches in the CESR ring, and the (b) asymmetry factor as a function of current.

Several observations can be made from the results: 1) between the currents from 1 to $13 \mathrm{~mA}$ per bunch, the electron bunch length increases by $6.0 \%$. This steady bunch length increase is not accompanied by a steady increase in the Gaussian asymmetry factor. 2) The single electron bunch length at low current is expected to be

* Work supported by the National Science Foundation.

+ Email: RLH@CESR10.LNS.Cornell.edu 
$\sigma_{z}=17.30 \mathrm{~mm}$ according to the CESR model. The measured single bunch length at low current was $\sigma_{z}=17.89 \pm 0.35 \mathrm{~mm}$, which is close to the multibunch measurement at low current of $\sigma_{z}=18.10 \pm 0.38 \mathrm{~mm}$ [2]. 3) With the bunch spacing of 42 ns between bunches, the longitudinal instability was not present. The threshold for the instability is $\sim 240 \mathrm{~mA}$ for this bunch spacing. A single data acquisition of the longitudinal bunch distribution for each of two different current settings is plotted in Figures 3 (a) and (c).
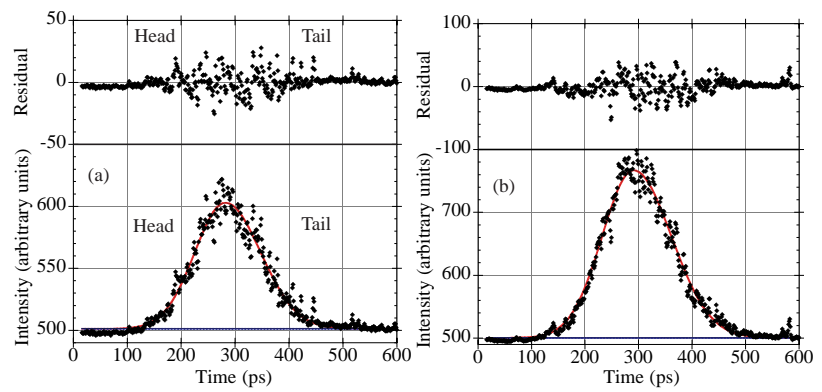

Figure 3. Single streak camera pictures of the CESR electron longitudinal bunch distribution and residuals at the current settings of: (a) $1.3 \mathrm{~mA}$ and (b) $12.5 \mathrm{~mA}$ per bunch.

The bunch length was measured as a function of the RF accelerating voltage. The measurements were made with electrons at each RF voltage, while keeping the bunch current constant. The measurement was made at a high current, and low current, with nine trains of two bunches separated by $42 \mathrm{~ns}$. The mean bunch width and root mean error is plotted at each RF voltage in Figures 4 (a) through (b).
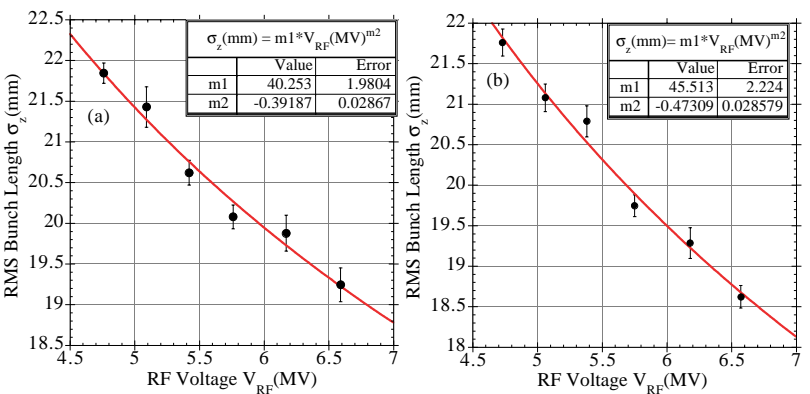

Figure 4. The electron bunch length as a function of RF accelerating voltage, with 18 bunches in the CESR Ring, at a current of (a) $9.0 \mathrm{~mA}$ per bunch and (b) $3.2 \mathrm{~mA}$ per bunch.

Fitting the data to the equation $\sigma\left(V_{r f}\right)=A V_{r f}^{m}$ the electron bunch lengths' dependence on the RF accelerating voltage was determined. In the low current case, $m=-0.47 \pm 0.03$, and in the high current case, $m=-0.39 \pm 0.03$. The expected value was $m=-0.50$. This is in good agreement with the measured value in the low current case. As the current increases, the bunch length dependence on RF voltage decreases, most likely due to beam loading in the RF cavities.
The bunch distribution was measured for both electrons and positrons, as a function of current, during high-energy collisions. The bunch spacing was $42 \mathrm{~ns}$. Each streak camera picture of the bunch distribution was fit to an asymmetric Gaussian distribution, and the width and asymmetry factor were plotted as a function of the current in Figures 5 (a) and (b).
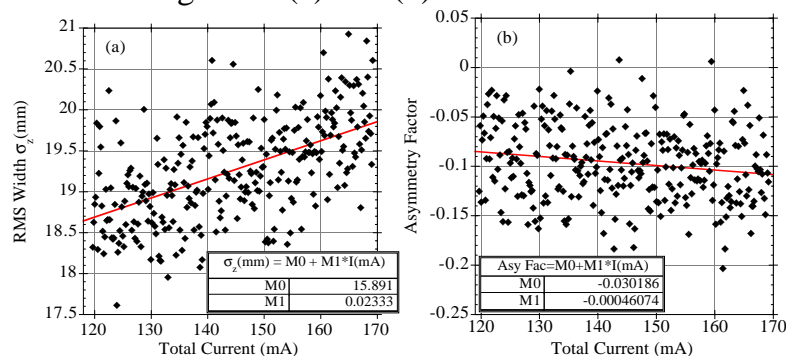

Figure 5. The electron (a) bunch length and (b) asymmetry factor, as a function of current, during highenergy collisions in CESR. The fit to the data is linear and points out the slow decrease in the bunch length and asymmetry factor as a function of current.

The bunch length, for both the electrons and positrons, decreases as a function of current. At the time of these measurements the total peak current during highenergy physics was $170 \mathrm{~mA}$ per beam. The runs lasted about 70 minutes, or until the single beam current was $120 \mathrm{~mA}$. Then CESR was refilled. The asymmetry factor, for both electrons and positrons, decrease as a function of current. Overall the electrons and positrons exhibit the same behavior in CESR.

\section{UNSTABLE BUNCH MOTION}

The CESR longitudinal instability is a dipole-coupled bunch instability, and it is only observed with multiple bunches per train. The characteristics of the instability are described elsewhere [3]. The threshold of the instability depends upon the mode of operation. We observed that: 1) at the instability threshold current, a signal at $f_{r} \pm f_{s}$ appears in the beams spectra, which indicates a predominately dipole structure in phase space. 2) As the current is increased, well above the threshold, the signal at $f_{r} \pm 2 f_{s}$ appears. 3) The threshold current for the instability is dependent on the bunch spacing in the train. 4) The instability degrades the CESR luminosity.

Measurements of the longitudinal instability were made at several different bunch spacings. They all have the same characteristics, except for different instability current thresholds. The one presented here is with nine trains of two bunches, with 14 ns spacing between bunches. The instability threshold with this bunch spacing was approximately $110 \mathrm{~mA}$. Just above the instability threshold, measurements of the electron bunch distribution were taken with the streak camera; both with the feedback on (no instability present), and with the longitudinal feedback off (the inability is present). A spectrum analyzer was used, in conjunction with the streak camera, to measure of the longitudinal instabilities presence.

The features of the longitudinal instability from the measurement are the following: 1) The dipole mode is not 
detectable by the streak camera (Figure 6). The bunch length and asymmetry factor, with and without the instability present, are in agreement (Table 1). 2) No structure is detected in the residuals of each of the data acquisitions.
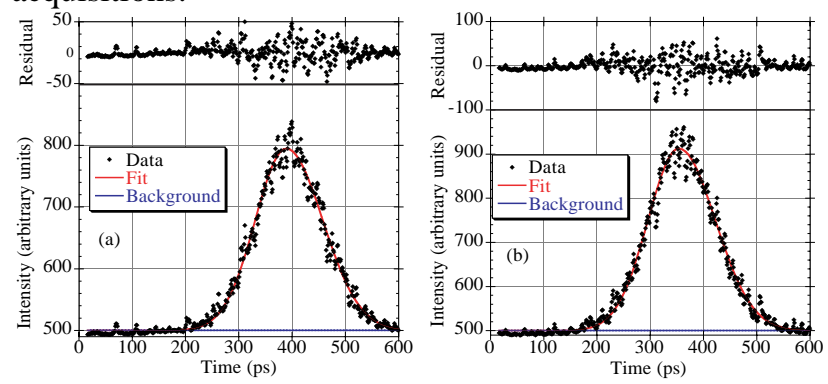

Figure 6. The CESR electron longitudinal bunch distribution measured by the streak camera when (a) the longitudinal instability is present and (b) the longitudinal instability is not present.

\begin{tabular}{|c|c|c|}
\hline & Stable Beam & Unstable Beam \\
\hline RMS Width $\sigma_{z}(\mathrm{~mm})$ & $18.78 \pm 0.13$ & $18.73 \pm 0.09$ \\
\hline Asymmetry Factor & $-0.07 \pm 0.01$ & $-0.07 \pm 0.01$ \\
\hline
\end{tabular}

Table 1. The measured electron bunch length and asymmetry factor for a stable and unstable beam in CESR.

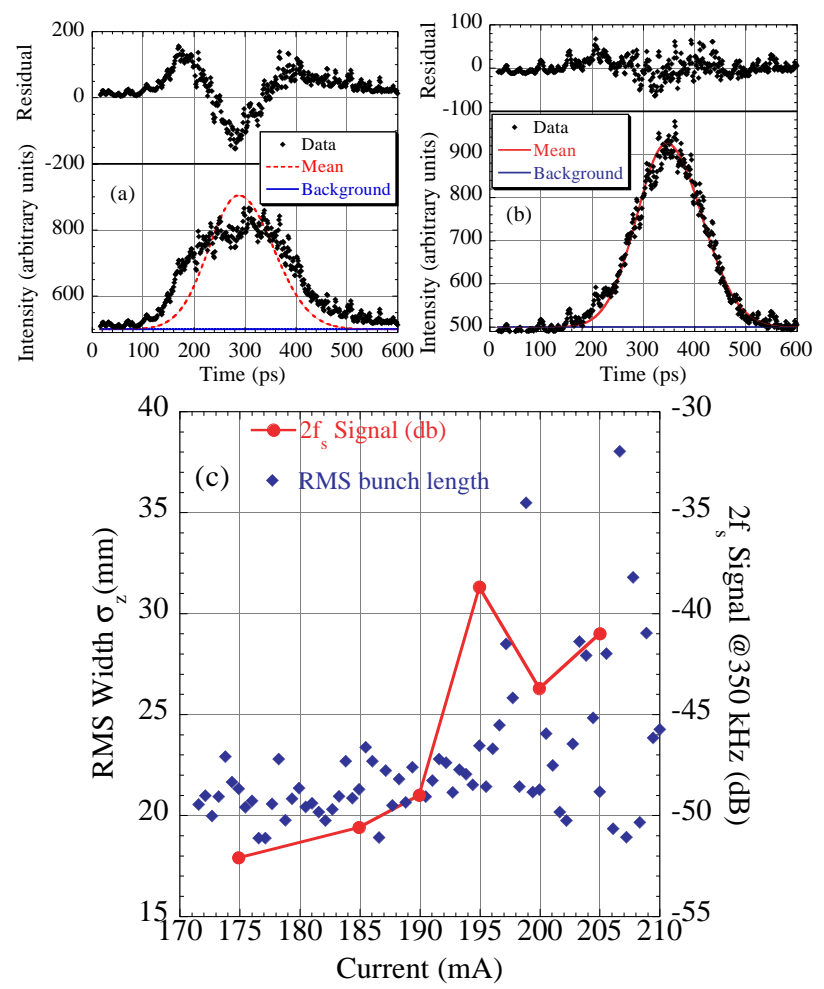

Figure 7. (a)-(b) The CESR bunch distribution fit to the mean stable bunch distribution when the beam is unstable ( $f_{s}$ and $2 f_{s}$ ) with the current above $200 \mathrm{~mA}$. The residuals are above the distribution. (c) The bunch length and $2 f_{s}$ signal amplitude as a function of current.

Exploring the instability behavior further, the current was increased and several interesting features appeared: 1) there is a steady increase in the bunch length as a function of current. Bunch lengthening increases dramatically, and the beam oscillates in size (signature of the quadrupole mode). 2) As the current increases, the $f_{r} \pm 2 f_{s}$ signal in the spectra appears, and it grows in strength as a function of current. The $2 f_{s}$ signal is plotted in Figure 7 (c) as a function of current. There is a dramatic jump in the signal amplitude, and the bunch distribution changes dramatically. Above $195 \mathrm{~mA}$ the bunch distribution oscillates violently. Figures 7 (a) and (b) are bunch distributions above $200 \mathrm{~mA}$. There is little evidence of the quadrupole mode in Figure 7 (b) and strong evidence in Figure 7 (a).

\section{CONCLUSIONS}

The measurements of CESR provided information about multibunch operations, as well as the behavior of the bunch, when the coupled-bunch longitudinal instability was present in CESR. The general conclusions from the measurements are that there is no difference between the positrons and electrons in CESR, in terms of longitudinal dynamics. The coupled-bunch longitudinal instability is a dipole mode instability, and a strong feedback system has allowed the instability to be damped, and increased the current thresholds [4]. The presence of the longitudinal instability was noticed in the beam spectra. It was also noted with the streak camera, but only when the quadrupole mode was present.

These studies of the longitudinal dynamics in CESR helped quantify some effects, such as: 1) the bunch length and asymmetry of the bunch increases as a function of current while colliding beams. 2) The bunch length increases as a function of current with multiple bunches in CESR. 3) The coupled bunch longitudinal instability is a dipole instability, but when the current is increased, it incites the quadrupole instability, and the bunch distribution changes drastically. Nonlinearites in the potential well and the large amplitude motion, in the dipole mode, give rise to the quadrupole mode. With the present feedback system, the coupled bunch instability is eliminated at currents above threshold for the quadrupole mode.

The streak camera has been invaluable as a diagnostic device for the measurement of the longitudinal parameters of CESR.

\section{ACKNOWLEDGMENTS}

The authors would like to thank the Stanford Linear Accelerator Center for the loan of the streak camera, especially Robert Siemann and Boris Podobedov.

\section{REFERENCES}

[1] Belomestnykh, S., Billing M., "Observation of a Longitudinal Coupled Bunch Instability in CESR", this proceeding.

[2] Holtzapple, R.L., et al, "Single Bunch Longitudinal Measurements at the Cornell Electron-Positron Storage Ring," CBN 98-23.

[3] Billing, M., "Observation of a Longitudinal Coupled Bunch Instability with Trains of Bunches in CESR," CLNS 98/1564.

[4] Sikora, J., et al, "Longitudinal Feedback at CESR", this proceeding. 